\title{
Comparison of real-time ultrasound imaging for manual lymphatic drainage on breast cancer-related lymphedema in individuals with breast cancer: a preliminary study
}

\author{
Dongkwon Seo ${ }^{a(i)}$, Seungwon Lee ${ }^{b, c(1)}$, Wonjae Choi $^{\mathrm{c}(\mathbb{C}}$ \\ ${ }^{a}$ Department of Physical Therapy, College of Medical Science, Konyang University, Daejeon, Republic of Korea \\ ${ }^{b}$ Department of Physical Therapy, College of Health Science and Social Welfare, Sahmyook University, Seoul, Republic of Korea \\ ${ }^{\mathrm{c} I n s t i t u t e}$ of SMART Rehabilitation, Sahmyook University, Seoul, Republic of Korea
}

\begin{abstract}
Objective: Breast cancer-related lymphedema (BCRL) is a major sequela after surgery or radiotherarpy for breast cancer. Manual lymphatic drainage (MLD) is designed to reduce lymph swelling by facilitating lymphatic drainage. This study attempted to determine the histologic changes in the skin and subcutaneous layer, and the immediate effect of MLD in decreasing lymphedema using ultrasound imaging, which is the method used most commonly to eliminate BCRL.

Design: A single-group experimental study.

Methods: Five subjects who were diagnosed with hemiparetic upper extremity lymphedema more than six months after breast cancer surgery participated in the study. MLD was performed for 60 minutes in the order of the thorax, breast, axilla, and upper arm of the affected side. In order to determine the effect of MLD, ultrasound imaging and limb volume were assessed. Two measurement tools were used for asessing lymphedema thickness among the pretest, posttest, and 30-minute follow-up period.

Results: Significant diferences in ultrasound imaging and upper limb volume were found between the affected side and non-affected side $(p<0.05)$. On the affected side, although ultrasound imaging showed a significant decrease after MLD $(p<0.05)$, there were no significant difference in upper limb volume when compared to the baseline.

Conclusions: In this study, a significant decrease in lymphedema by MLD was demonstrated by ultrasound imaging, which is considered to be more useful in assessing histological changes than limb volume measurements. Further research on the protocol for eliminating lymphedema will be needed.
\end{abstract}

Key Words: Breast cancer lymphedema, Manual lymphatic drainage, Ultrasonography

\section{Introduction}

Breast cancer-related lymphedema (BCRL) is a major side effect and a complication from breast cancer surgery and radiotherapy, causing chronic lymphedema in the arm [1]. Normal lymphatic drainage plays a key role in the elimination and transfer of waste matter from the body (bilirubin, pigments), and ultimately prevents swelling [2]. Individuals with BCRL have a malfunctioning lymphatic system, which delays lymphatic drainage, causing an abnormal increase of tissue protein and resulting in chronic inflammation, fibrosis, pain, limited range of motion, or paraesthesia $[3,4]$. This leads to functional disabilities and mental problems, such as anxiety and depression [5,6]. Areas with BCRL are reported to have decreased local immune function, result in having a higher risk of infection and inflammation. Therefore, appropriate interventions need to be applied to reduce and prevent BCRL [4,7].

BCRL develops between the skin and subcutaneous layer and this has been proven by various soft tissue imaging as-

Received: 13 December, 2019 Revised: 3 March, 2020 Accepted: 3 March, 2020

Corresponding author: Wonjae Choi (ORCID https://orcid.org/0000-0002-2232-6744)

Institute of SMART Rehabilitation, Sahmyook University, 815 Hwarang-ro, Nowon-gu, Seoul 01795, Republic of Korea

Tel: 82-2-3399-1630 Fax: 82-2-3399-1639 E-mail: sadman5@naver.com

(c) This is an Open-Access article distributed under the terms of the Creative Commons Attribution Non-Commercial License (http://creativecommons.org/licenses/ by-nc/4.0) which permits unrestricted non-commercial use, distribution, and reproduction in any medium, provided the original work is properly cited.

Copyright $(\odot 2020$ Korean Academy of Physical Therapy Rehabilitation Science 
sessment methods, including magnetic resonance imaging, computed tomography, and ultrasound imaging in soft tissue imaging [8,9]. A recent study comparing magnetic resonance imaging and ultrasound imaging reported that ultrasound imaging does not provide clear images of tissue composition compared to magnetic resonance imaging. However, it can differentiate any histologic changes of the skin and subcutaneous layer due to lymphedema, check for decreases in lymphedema after complex decongestive therapy (CDT), and evaluate tissue thickness and consistency [10]. This will play an important role in evaluation of the treatment result. Ultrasound imaging is safe from radiologic dangers, easy to use, saves time and cost, and uses a non-invasive technique $[11,12]$.

CDT is a conventional method for management of BCRL. CDT is composed of manual lymphatic drainage (MLD), bandaging, lymphatic exercise, skin care, and education on self-management. In Europe, a traditional CDT is divided into two phases. In the primary phase, lymphedema size is decreased, subcutaneous tissue and extremities are restored, and healthy skin is maintained [13]. In the secondary phase, the goal is to maintain what has been gained from phase I [14]. MLD plays an important role in CDT. It is recommended for maintenance of MLD with simple lymphatic drainage and self-administered massage, although they are not performed by professionals [15].

MLD could promote lymphatic function, remove lymphedema between the skin and the subcutaneous layer, decrease fibrosis of skin tissue, and restore skin health [16]. Previous studies have reported on the effect of MLD in decreasing lymphedema $[15,16]$. Lymphedema can be decreased and secondary lymphedema can be prevented when physical therapy with MLD is performed early [3]. Traditional evaluation of lymphedema decreased by MLD is performed with volume measurements, such as tape measurements, perometry, and water displacement [17]. These methods only measure skin volume and cannot assess histologic decreases in lymphedema between the skin and subcutaneous layer. However, ultrasound imaging is a useful tool for evaluating changes in lymphedema because it is an easy, fast, and safe diagnostic method [3,18]. A previous study that used ultrasound imaging only measured and compared the thickness of lymphedema in the non-affected side and affected side of the subjects but no study has proven the histologic changes between the skin and subcutaneous layer when MLD is used to decrease lymphedema [11]. Therefore, the aim of this study was to determine the histologic changes in the skin and subcutaneous layer using ultrasound imaging as well as the immediate effect of MLD in decreasing lymphedema.

\section{Methods}

\section{Participants and procedure}

This study is a single-group experimental study design. This study recruited six women who underwent breast cancer surgery and were diagnosed with upper extremity lymphedema at Konyang University Hospital by the convenience sampling method. The inclusion criteria of this study was individuals with hemiparetic upper extremity lymphedema for more than six months after breast cancer surgery and with $10 \%$ greater volume of lymphedema in the affected side. The exclusion criteria was individuals with peripheral nerve injury, drug intoxication, nephritic syndrome, those who use diuretics or other edema treatments. Those who had understood the purpose and procedures of this study and had signed consent forms participated in the study. This study was approved from the Institutional Review Board of Sahmyook University (IRB No. SYUIRB2012-003). The baseline was measured before intervention, and a posttest was performed to confirm the immediate effects after MLD of 60 minutes. A follow-up test was conducted after 30 minutes to confirm the prolonged effect of MLD [19]. Ultrasonography and limb volume were measured to investigate for reduction of lymphedema in participants.

\section{Intervention}

In order to determine the effect of MLD, subjects were instructed to halt all other treatment for 24 hours. As a method for elimination of BCRL and for the promotion of lymph drainage, removal of tissue protein and highly embedded tissue fluid, soft massage of MLD, as suggested by Dr. Vodder, was used, and the following areas were massaged [20]: Thorax; breast; axilla; and upper arm of the affected side. Three therapists performed MLD; all of the therapists had completed MLD education, and had more than two years of clinical experience. Two therapists treated two subjects each, and the third therapist treated one subject for $60 \mathrm{mi}-$ nutes each. The preset MLD program was used in order to minimize the difference between therapists and to increase objectivity of MLD application in order to ensure use of the same method of MLD in terms of order and time. 


\section{Outcome measurements}

Subjects were tested while seated on a chair with armrests with the forearm supinated and relaxed. An ultrasound unit (MySono U5; Samsung Medison Co., Seoul, Korea) measured the upper arm and forearm using a $7.5 \mathrm{MHz}$ linear transducer. Pre-intervention, post-intervention, and followup periods were measured separately; in pre-intervention both the non-affected side and the affected side were measured and compared, and for post-intervention and followup, only the affected side was measured in order to check the effect of MLD. Each measurement was repeated three times and the average was used. A transducer was placed $10 \mathrm{~cm}$ proximal to the elbow crease along the line between the midpoint of the medial and lateral epicondyles of the humerus and the bicipital groove. On the forearm, it was placed $10 \mathrm{~cm}$ distal from the elbow crease along the line between the midline and lateral epicondyles and the midpoint of the radial and ulnar styloid processes. Ultrasound imaging used the maximal compression method, and this study used the sum of the skin and subcutaneous layer for lymphedema thickness (Figure 1) [18].

Limb volume measurements were performed in the same position as the ultrasound imaging measurement, with the same measurement location; a ruler was used for repeated measurements, performed three times. Measurements were performed at pre-intervention, post-intervention, and follow-up periods. However, volume measurements were performed by a different person than that for ultrasound imaging measurement.

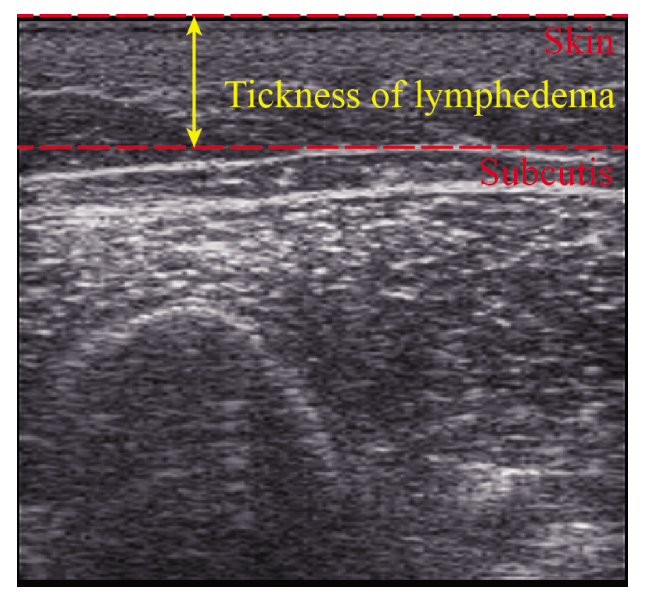

Figure 1. The thickness of breast cancer-related lymphedema. Yellow arrow indicated ultrasound images of lymphedema demonstrating the thickness of the skin, subcutis tissue, and their sum with maximal compression.

\section{Data analysis}

The collected data were analysed using SPSS Statistics for Windows, Version 15.0 (SPSS Inc., Chicago, IL, USA). The Mann-Whitney U-test was used for comparison of lymphedema thickness between the non-affected and the affected sides. The repeated measures ANOVA was used for the three repeated measurements of post-MLD effect in the affected side. Bonferoni was used for pre, post, and followup analysis. The Bonferroni post hoc was applied to adjust the statistical significance level to 0.05 .

\section{Results}

The results from five subjects, excluding one without edema, were obtained. General characteristics such as age, height, weight, injury sites, and cancer stages are shown in Table 1.

Ultrasound imaging measurement and limb volume measurement results showed significant differences in lymphedema thickness between the non-affected side and the affected side. Volume was increased in the affected side, compared with the non-affected side, and lymphedema thickness was increased between skin and subcutaneous layer $(p<$ $0.05)$. However, after application of MLD to decrease lymphedema on the affected side, the repeated measurements of ultrasound imaging measurement and limb volume measurements were different. In the repeated measurements during the pre, post, and follow-up periods, ultrasound imaging measurements showed a significant decrease $(p<0.05)$, however, limb volume measurements did not show a significant decrease (Figure 2).

\section{Discussion}

In this study, lymphedema thickness was measured using the maximal compression method suggested by a previous

Table 1. General characteristics of participants

\begin{tabular}{lc}
\multicolumn{1}{c}{ Characteristics } & Value \\
\hline Age $(\mathrm{y})$ & $47.60(11.22)$ \\
Height $(\mathrm{cm})$ & $155.60(7.96)$ \\
Affected side (left/right) & $2 / 3$ \\
Cancer stage (stage 3/4) & $4 / 1$ \\
Weight (kg) & $63.40(5.46)$ \\
Duration since initial cancer therapy & $49.80(33.92)$ \\
\hline
\end{tabular}

Values are presented as mean (SD). 

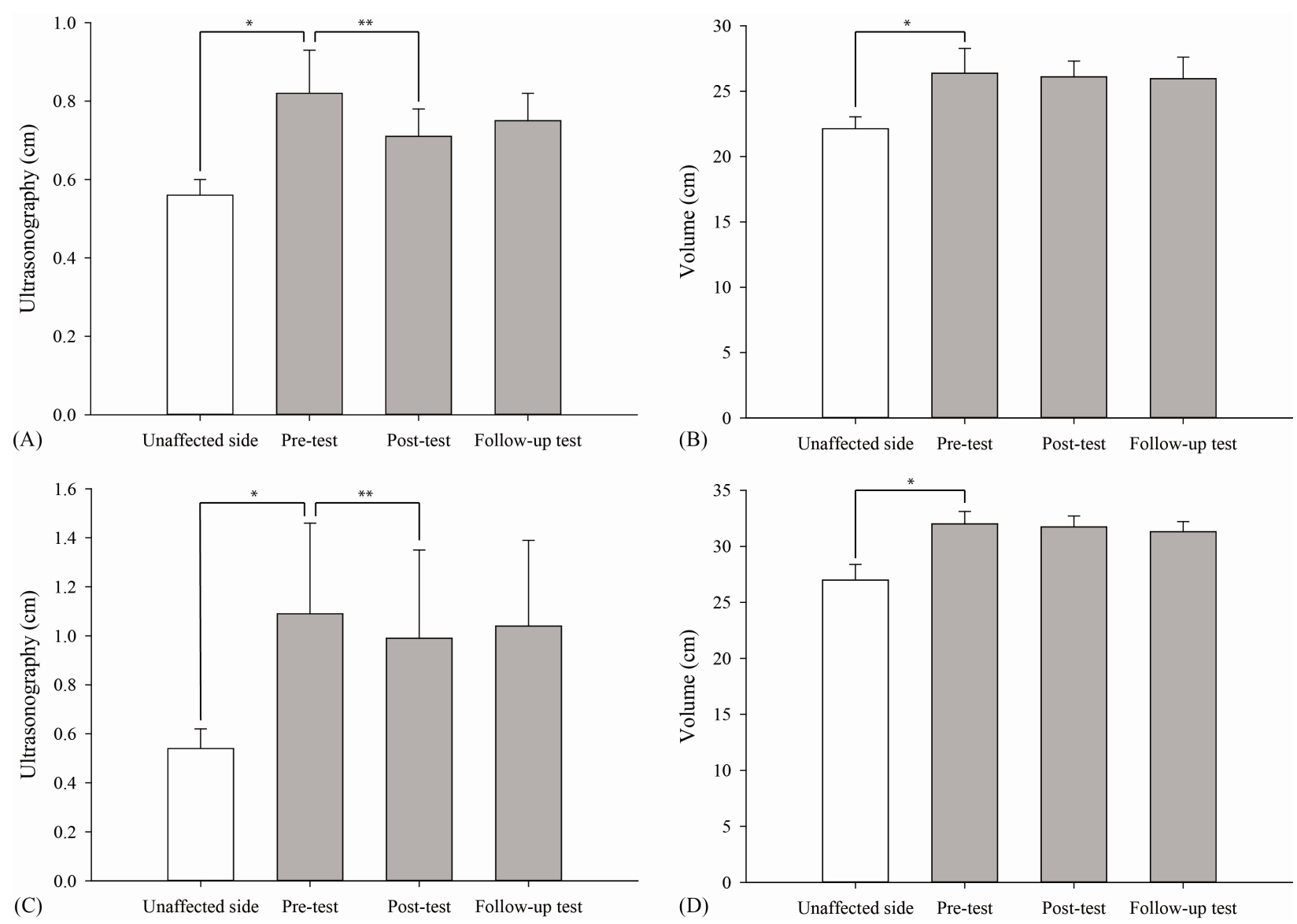

Figure 2. The thickness change of lymphedema in each test. (A) Ultrasonography of below elbow, (B) volume of below elbow, (C) ultrasonography of above elbow, and (D) volume of above elbow. The white bar is unaffected side and the gray bar is affected side. ${ }^{*} p<0.05$, $* * p<0.017$.

study [18]. Use of the maximal compression method can allow for attainment of more accurate measurement values because ultrasound imaging is evenly distributed, and because ultrasound imaging is not affected by demographic and clinical data (age, body weight, and metastasis), and therefore making it possible to obtain objective measurements. In this study, the maximal compression method was used for objective and accurate evaluation. In a pilot test, use of the maximal compression method showed no pain or discomfort. Therefore, ultrasound imaging measurement was selected for use in this study. Limb volume measurement was performed using a ruler. Use of a ruler is common for measurement of edema, and this method has been used in many previous studies. In this study, volume measurements were compared with ultrasound imaging.

In this study, MLD was used to decrease edema in individuals with lymphedema. This method was the one most commonly used, and was performed by a therapist. MLD performed by a therapist was more effective than self-massage in reducing edema [16], and more effective when CDT was applied with MLD [21]. MLD is more effective when applied with other compression methods, such as garment and bandaging, pneumatic compression, and limb exercise, in order to decrease lymphedema $[15,22]$. MLD was studied because of its effects in decreasing lymphedema. MLD was used in this study because it is a therapeutic method that provides various benefits to patients, and is applied by therapists.

Previous studies have reported on the effectiveness of MLD in decreasing edema, however, checking the difference between arm circumference and volume measurement, and the decrease in lymphedema between skin and subcutaneous layer can be difficult. Computed tomography and magnetic resonance imaging have been used in previous studies for evaluation of the therapeutic effect of lymphedema or decreases in edema, but because they are harmful, continuous evaluation of the changes could not be performed. 
However, a recent study used ultrasound imaging to prove the therapeutic effects of lymphedema between skin and subcutaneous layer [11]. Therefore, this study used ultrasound imaging to measure the lymphedema decrease in the skin and subcutaneous layer. In addition, in order to check the result after MLD on ultrasound images, the measurement was performed immediately after MLD, and was followed up for evaluation for any continued effect.

The results of this study showed a significant decrease in ultrasound imaging measurement after MLD; however, limb volume measurement did not show a significant decrease. Application of MLD on individuals with lymphedema resulted in decreased edema in the skin and subcutaneous layer, and showed a continued effect. The results of this study support the results from previous studies showing that MLD is effective in decreasing lymphedema $[3,11,23]$. However, in this study, decreased lymphedema in the skin and subcutaneous layer by MLD was observed through ultrasound imaging. The findings of this study suggest that ultrasound imaging monitors for changes in lymphedema in real time, and that it can be used clinically. According to this result, real-time ultrasound imaging can be used clinically in order to visibly evaluate changes in patients receiving MLD. Limb volume measurements did not show a significant decrease, however, statistical numbers showed a continuous decrease. Therefore, the results of this study showed that the decrease in volume measurement took place in the skin and subcutaneous layer first, and then in the volume later.

In this study, the effect of MLD on decreasing lymphedema in the skin and subcutaneous layer was proven by ultrasound imaging. With ultrasound imaging, this study demonstrated the possibility of using real-time ultrasound images to show objective data to patients. MLD should be used more actively to decrease lymphedema and to show the treatment effect by real-time ultrasound imaging.

The limitation of this study was designed by a singlegroup experimental study and the small number of subjects, thereby limiting the ability to generalize the results. Cancer patients had less participation due to decreased social activities because of depression and mental disorders. Conduct of additional studies of cancer patients will be needed in the future. In ultrasound imaging, the follow-up measurement increased volume. This study was not good in predicting tissue changes. Among CDTs, only MLD was selected, and other treatment methods were not compared. Therefore, more CDT methods using ultrasound imaging for comparison of lymphedema in the skin and subcutaneous layer are needed.

\section{Acknowledgements}

This paper was supported by the Fund of Konyang University and Sahmyook University, and the authors are grateful to N.Y Jeon, and A. Kim for their contributions to the recruitment of the subjects.

\section{Conflict of Interest}

The authors declared no potential conflicts of interest with respect to the authorship and/or publication of this article.

\section{References}

1. Shah C, Vicini FA. Breast cancer-related arm lymphedema: incidence rates, diagnostic techniques, optimal management and risk reduction strategies. Int J Radiat Oncol Biol Phys 2011;81: 907-14.

2. Bahtiyarca ZT, Can A, Ekşioğlu E, Çakcı A. The addition of selflymphatic drainage to compression therapy instead of manual lymphatic drainage in the first phase of complex decongestive therapy for treatment of breast cancer-related lymphedema: a randomized-controlled, prospective study. Turk J Phys Med Rehabil 2019;65:309-17.

3. Torres Lacomba M, Yuste Sánchez MJ, Zapico Goñi A, Prieto Merino D, Mayoral del Moral O, Cerezo Téllez E, et al. Effectiveness of early physiotherapy to prevent lymphoedema after surgery for breast cancer: randomised, single blinded, clinical trial. BMJ 2010;340:b5396.

4. Gatt M, Willis S, Leuschner S. A meta-analysis of the effectiveness and safety of kinesiology taping in the management of cancer-related lymphoedema. Eur J Cancer Care (Engl) 2017. doi: 10.1111/ecc.12510. [Epub ahead of print]

5. Tsang RY, Mackey JR. Delayed resolution of jaundice in the arm of a patient with impaired lymphatic drainage: a case report. Clin Breast Cancer 2007;7:890-1.

6. Ergin G, Şahinoğlu E, Karadibak D, Yavuzşen T. Effectiveness of Kinesio Taping on anastomotic regions in patients with breast cancer-related lymphedema: a randomized controlled pilot study. Lymphat Res Biol 2019;17:655-60.

7. Mortimer PS. Managing lymphedema. Clin Dermatol 1995;13: 499-505.

8. Testoni PA, Bagnolo F, Andriulli A, Bernasconi G, Crotta S, Lella F, et al. Octreotide 24-h prophylaxis in patients at high risk for post-ERCP pancreatitis: results of a multicenter, randomized, controlled trial. Aliment Pharmacol Ther 2001;15:965-72.

9. Deltombe T, Jamart J, Recloux S, Legrand C, Vandenbroeck N, Theys S, et al. Reliability and limits of agreement of circumferential, water displacement, and optoelectronic volumetry in the measurement of upper limb lymphedema. Lymphology 2007; 40:26-34. 
10. O'Donnell TF Jr, Rasmussen JC, Sevick-Muraca EM. New diagnostic modalities in the evaluation of lymphedema. J Vasc Surg Venous Lymphat Disord 2017;5:261-73.

11. Tassenoy A, De Mey J, De Ridder F, Van Schuerbeeck P, Vanderhasselt T, Lamote J, et al. Postmastectomy lymphoedema: different patterns of fluid distribution visualised by ultrasound imaging compared with magnetic resonance imaging. Physiotherapy $2011 ; 97: 234-43$.

12. Sezgin Ozcan D, Oken O, Dalyan Aras M, Koseoglu BF. Is ultrasonography a useful method to evaluate the effectiveness of complex decongestive therapy in breast cancer-related lymphedema? Lymphology 2017;50:84-94.

13. Cohen SR, Payne DK, Tunkel RS. Lymphedema: strategies for management. Cancer 2001;92(4 Suppl):980-7.

14. Földi E. The treatment of lymphedema. Cancer 1998;83(12 Suppl American):2833-4.

15. Ezzo J, Manheimer E, McNeely ML, Howell DM, Weiss R, Johansson KI, et al. Manual lymphatic drainage for lymphedema following breast cancer treatment. Cochrane Database Syst Rev 2015;(5):CD003475.

16. Shao Y, Zhong DS. Manual lymphatic drainage for breast cancer-related lymphoedema. Eur J Cancer Care (Engl) 2017. doi: 10.1111/ecc.12517. [Epub ahead of print]
17. Sharif S, Brennan P, Rawluk D. Non-surgical treatment of meningioma: a case report and review. Br J Neurosurg 1998; 12:36972 .

18. Lim CY, Seo HG, Kim K, Chung SG, Seo KS. Measurement of lymphedema using ultrasonography with the compression method. Lymphology 2011;44:72-81.

19. Guerero RM, das Neves LMS, Guirro RRJ, Guirro ECO. Manual lymphatic drainage in blood circulation of upper limb with lymphedema after breast cancer surgery. J Manipulative Physiol Ther 2017;40:246-9.

20. Kasseroller RG. The Vodder School: the Vodder method. Cancer 1998;83(12 Suppl American):2840-2.

21. Devoogdt N, Christiaens MR, Geraerts I, Truijen S, Smeets A, Leunen $\mathrm{K}$, et al. Effect of manual lymph drainage in addition to guidelines and exercise therapy on arm lymphoedema related to breast cancer: randomised controlled trial. BMJ 2011;343:d5326.

22. Gillespie TC, Sayegh HE, Brunelle CL, Daniell KM, Taghian AG. Breast cancer-related lymphedema: risk factors, precautionary measures, and treatments. Gland Surg 2018;7:379-403.

23. Koul R, Dufan T, Russell C, Guenther W, Nugent Z, Sun X, et al. Efficacy of complete decongestive therapy and manual lymphatic drainage on treatment-related lymphedema in breast cancer. Int J Radiat Oncol Biol Phys 2007;67:841-6. 